\title{
Monogenic functions in commutative algebras associated with classical equations of mathematical physics
}

\author{
Sergiy A. Plaksa \\ Dedicated to memory of my colleagues \\ Igor Mel'nichenko and Volodymyr Kovalev \\ on the occasion of their 80th birthday \\ (Presented by V. Ya. Gutlyanskii)
}

\begin{abstract}
Analytic function methods in the complex plane for plane potential fields inspire searching analogous effective methods for solving spatial and multidimensional problems of mathematical physics. Many such methods are based on mappings of hypercomplex algebras.

An idea of an algebraic-analytic approach to elliptic equations of mathematical physics means a finding of commutative Banach algebra such that differentiable functions with values in this algebra have components satisfying the given equation with partial derivatives. An use of differentiable functions given in commutative Banach algebras combines the preservation of basic properties of analytic functions of a complex variable for the mentioned differentiable functions and the convenience and the simplicity of construction of solutions of PDEs.

The paper contains the review of results reflecting the formation and the development of the mentioned approach.
\end{abstract}

2010 MSC. 30G35, 35J05, 31A30.

Key words and phrases. Laplace equation, biharmonic equation, axial-symmetric potential, Stokes flow function, commutative Banach algebra, monogenic function, analytic function, Cauchy-Riemann conditions, Cauchy integral theorem, Cauchy integral formula, Morera theorem, Taylor expansion, Laurent expansion.

\section{Introduction}

Defining a stationary potential solenoid field in a simply connected domain of the three-dimensional real space $\mathbb{R}^{3}$, the vector-function $\mathbf{V}$

Received 20.12.2018 
satisfies the system of equations $\operatorname{div} \mathbf{V}=0, \operatorname{rot} \mathbf{V}=0$, that have the following expanded form:

$$
\begin{aligned}
& \frac{\partial v_{1}}{\partial x}+\frac{\partial v_{2}}{\partial y}+\frac{\partial v_{3}}{\partial z}=0, \\
& \frac{\partial v_{3}}{\partial y}-\frac{\partial v_{2}}{\partial z}=0, \quad \frac{\partial v_{1}}{\partial z}-\frac{\partial v_{3}}{\partial x}=0, \quad \frac{\partial v_{2}}{\partial x}-\frac{\partial v_{1}}{\partial y}=0,
\end{aligned}
$$

where $\mathbf{V}:=\left(v_{1}, v_{2}, v_{3}\right)$ and $v_{k}:=v_{k}(x, y, z)$ for $k=1,2,3$ are real scalar functions of Cartesian coordinates $x, y, z$.

For a potential solenoid field there exists a scalar potential function $u(x, y, z)$ such that $\mathbf{V}=\operatorname{grad} u:=\left(\frac{\partial u}{\partial x}, \frac{\partial u}{\partial y}, \frac{\partial u}{\partial z}\right)$, and $u$ satisfies the three-dimensional Laplace equation

$$
\Delta_{3} u(x, y, z):=\left(\frac{\partial^{2}}{\partial x^{2}}+\frac{\partial^{2}}{\partial y^{2}}+\frac{\partial^{2}}{\partial z^{2}}\right) u(x, y, z)=0 .
$$

It is evident that in the case of plane field, where $v_{3} \equiv 0$ and the functions $v_{1}, v_{2}$ do not depend on $z$, the system (1.1) turns into the classical Cauchy-Riemann conditions for components of complex potential $F(x+i y)=v_{1}(x, y)+i v_{2}(x, y)$ being an analytic function of complex variable $x+i y$. Moreover, every analytic function $F(x+i y)$ satisfies the two-dimensional Laplace equation

$$
\left(\frac{\partial^{2}}{\partial x^{2}}+\frac{\partial^{2}}{\partial y^{2}}\right) F(x+i y) \equiv F^{\prime \prime}(x+i y)\left(1^{2}+i^{2}\right)=0
$$

due to the equality $1^{2}+i^{2}=0$ for the unit 1 and the imaginary unit $i$ of the algebra of complex numbers.

Analytic function methods in the complex plane for plane potential fields inspire searching analogous effective methods for solving spatial and multidimensional problems of mathematical physics. Many such methods are based on mappings of hypercomplex algebras.

\section{Hypercomplex analysis: origination and initial applications}

\subsection{A noncommutative algebra of quaternions}

Apparently, W. Hamilton [22] made the first attempts to construct an algebra associated with the three-dimensional Laplace equation (1.2) in that sense that components of hypercomlex functions satisfy Eq. (1.2).

W. Hamilton [22] constructed a noncommutative algebra of quaternions with the basis $\{1, i, j, k\}$ and the multiplication table

$$
i^{2}=j^{2}=k^{2}=-1, \quad i j=-j i=k, \quad j k=-k j=i, \quad k i=-i k=j .
$$


Using the operator $\nabla:=i \frac{\partial}{\partial x}+j \frac{\partial}{\partial y}+k \frac{\partial}{\partial z}$, he rewrote Eqs. (1.1) in an equivalent form $\nabla W(x, y, z)=0$ for the function $W(x, y, z):=$ $i v_{1}(x, y, z)+j v_{2}(x, y, z)+k v_{3}(x, y, z)$. In this case Eqs. (1.1) can be considered as analogues of Cauchy - Riemann conditions for the function $W(x, y, z)$ taking values in the algebra of quaternions.

Gr. Moisil and N. Théodoresco [47], R. Fueter [7] considered generalizations of system (1.1) for functions taking values in the algebra of quaternions.

Nevertheless, in spite of convenience to write compactly many relations for potential fields in quaternion form, during a long time, the development of applications of quaternion-valued functions was restrained by undevelopment of methods for effective construction of such functions as well as functions taking values in others noncommutative algebras.

Last decades, one can see the essential progress in the theory of mappings of noncommutative algebras and their applications to problems of mathematical physics (see, e.g., the papers of A. Sudbery [99], K. Gürlebeck and W. Spröessig [20], V. Kravchenko and M. Shapiro [32], H. Leutwiler [36], J. Ryan [80], F. Colombo, I. Sabadini and D. Struppa [5] and many other papers).

\subsection{Description of low-dimensional commutative algebras}

After Hamilton's discovery of algebra of quaternions the active constructions of algebras of hypercomplex numbers began.

In particular, to describe linear associative algebras, B. Peirce [49] has introduced concepts of nilpotent and idempotent elements and has constructed multiplication tables for 163 algebras of dimensionality not greater 6 , but has considered not all three-dimensional and four-dimensional algebras.

E. Study [98] has described all associative algebras with unit up to the fourth dimension inclusive over the field of real or complex numbers. It follows from results of the paper [98] that there exist only 2 two-dimensional commutative associative algebras with unit 1 over the complex field $\mathbb{C}$ (let us denote their by $\mathbb{B}_{0}$ and $\mathbb{B}$ ) and 4 three-dimensional algebras with the same properties (let us denote their by $\mathbb{A}_{1}, \mathbb{A}_{2}, \mathbb{A}_{3}$ and $\mathbb{A}_{4}$ ). If one chooses nilpotent and idempotent elements generating these algebras, then the multiplication tables will of the simplest form.

Let $\mathbb{B}_{0}$ be a semisimple algebra with idempotent elements in the basis $\left\{\mathcal{I}_{1}, \mathcal{I}_{2}\right\}$ and the multiplication table: $\mathcal{I}_{1}^{2}=\mathcal{I}_{1}, \mathcal{I}_{2}^{2}=\mathcal{I}_{2}, \mathcal{I}_{1} \mathcal{I}_{2}=0$. Here $1=\mathcal{I}_{1}+\mathcal{I}_{2}$.

The algebra $\mathbb{B}$ with the basis $\{1, \rho\}$, where $\rho^{2}=0$, contains the onedimensional radical generated by the element $\rho$. 
Let $\mathbb{A}_{1}$ be a semisimple algebra with idempotent elements in the basis $\left\{\mathcal{I}_{1}, \mathcal{I}_{2}, \mathcal{I}_{3}\right\}$ and the multiplication table: $\mathcal{I}_{1}^{2}=I_{1}, \mathcal{I}_{2}^{2}=\mathcal{I}_{2}, \mathcal{I}_{3}^{2}=\mathcal{I}_{3}$, $\mathcal{I}_{1} \mathcal{I}_{2}=\mathcal{I}_{1} \mathcal{I}_{3}=\mathcal{I}_{2} \mathcal{I}_{3}=0$. Here $1=\mathcal{I}_{1}+\mathcal{I}_{2}+\mathcal{I}_{3}$.

The algebras $\mathbb{A}_{2}, \mathbb{A}_{3}$ and $\mathbb{A}_{4}$ contain radicals.

Let $\mathbb{A}_{2}$ be an algebra with the basis $\left\{\mathcal{I}_{1}, \mathcal{I}_{2}, \rho\right\}$ and the multiplication table: $\mathcal{I}_{1}^{2}=\mathcal{I}_{1}, \mathcal{I}_{2}^{2}=\mathcal{I}_{2}, \mathcal{I}_{1} \mathcal{I}_{2}=0, \rho^{2}=0, \mathcal{I}_{1} \rho=0, \mathcal{I}_{2} \rho=\rho$. Here $1=\mathcal{I}_{1}+\mathcal{I}_{2}$, and $\rho$ belongs to the one-dimensional radical of algebra.

Algebras $\mathbb{A}_{3}$ and $\mathbb{A}_{4}$ have the basis $\left\{1, \rho_{1}, \rho_{2}\right\}$, where $\rho_{1}$ and $\rho_{2}$ belong to the radicals of these algebras. The multiplication table in the algebra $\mathbb{A}_{3}$ is of the form: $\rho_{1}^{2}=\rho_{2}, \rho_{2}^{2}=0, \rho_{1} \rho_{2}=0$, and the multiplication table in the algebra $\mathbb{A}_{4}$ is of the form: $\rho_{1}^{2}=\rho_{2}^{2}=\rho_{1} \rho_{2}=0$. Thus, algebras $\mathbb{A}_{3}$ and $\mathbb{A}_{4}$ have the two-dimensional radicals.

C. Segre [82] considered commutative algebras of multicomplex numbers. Such algebras are constructed by an inductive way and have dimensions $2^{n}$ as algebras over the real field $\mathbb{R}$. In particular, the algebra of bicomplex numbers (they are also called by the Segre quaternions) has the dimension 4 over the field $\mathbb{R}$ and is isomorphic to the mentioned algebra $\mathbb{B}_{0}$.

E. Cartan [4] proved that for any finite-dimensional associative algebra with unit there exist a basis composed of nilpotent and idempotent elements, and he specified the multiplication table for such a basis.

\subsection{Attempts to use commutative algebras for constructions of solutions of classical equations of mathematical physics}

The first attempts to use commutative algebras for constructions of solutions of three-dimensional Laplace equation gave often negative results.

Let $\mathbb{A}$ be a $n$-dimensional commutative associative Banach algebra with unit 1 over either the field $\mathbb{R}$ or the field $\mathbb{C}, 3 \leq n \leq \infty$. Let $\left\{e_{1}, e_{2}, e_{3}\right\}$ be a part of a basis of $\mathbb{A}$ and $E_{3}:=\left\{\zeta:=x e_{1}+y e_{2}+z e_{3}:\right.$ $x, y, z \in \mathbb{R}\}$ be the linear span generated by the vectors $e_{1}, e_{2}, e_{3}$.

Let us use the same denotation $\Omega$ for a domain $\Omega \subset \mathbb{R}^{3}$ and for the domain in $E_{3}$ that is congruent to the domain $\Omega$.

A function $\Phi: \Omega \longrightarrow \mathbb{A}$ is analytic in a domain $\Omega \subset E_{3}$ if in a certain neighborhood of every point $\zeta_{0} \in \Omega$ it can be represented in the form of the sum of convergent power series with coefficients belonging to the algebra $\mathbb{A}$.

H. A. v. Beckh-Widmanstetter [1] has proved that there does not exist a three-dimensional commutative associative algebra with unit $e_{1}=1$ over the field $\mathbb{R}$ such that all components of expansion of analytic function

$$
\Phi\left(x e_{1}+y e_{2}+z e_{3}\right)=u_{1}(x, y, z) e_{1}+u_{2}(x, y, z) e_{2}+u_{3}(x, y, z) e_{3}
$$


with respect to the basis $\left\{e_{1}, e_{2}, e_{3}\right\}$ satisfy $E q$. (1.2).

Nevertheless, P. W. Ketchum [26] used analytic functions with values in commutative algebras for a construction of solutions of threedimensional Laplace equation (1.2). He has shown that if linearly independent elements $e_{1}, e_{2}, e_{3} \in \mathbb{A}$ satisfy the condition

$$
e_{1}^{2}+e_{2}^{2}+e_{3}^{2}=0
$$

then every analytic function $\Phi(\zeta)$ of the variable $\zeta=x e_{1}+y e_{2}+z e_{3}$ satisfies Eq. (1.2) due to the relations

$$
\left(\frac{\partial^{2}}{\partial x^{2}}+\frac{\partial^{2}}{\partial y^{2}}+\frac{\partial^{2}}{\partial z^{2}}\right) \Phi(\zeta) \equiv \Phi^{\prime \prime}(\zeta)\left(e_{1}^{2}+e_{2}^{2}+e_{3}^{2}\right)=0,
$$

where $\Phi^{\prime \prime}:=\left(\Phi^{\prime}\right)^{\prime}$ and $\Phi^{\prime}$ is defined by the equality $d \Phi=\Phi^{\prime}(\zeta) d \zeta$ as well as in the paper of G. Scheffers [81].

P. W. Ketchum [26] called an algebra $\mathbb{A}$ harmonic if in $\mathbb{A}$ there exists a triad of linearly independent vectors satisfying the equality (2.1). We shall say that such a triad $\left\{e_{1}, e_{2}, e_{3}\right\}$ is also harmonic. Later I.P. Mel'nichenko [42] added the requirement $e_{k}^{2} \neq 0$ for $k=1,2,3$ to exclude from a consideration trivial triads kind of $e_{1}=1, e_{2}=i$ and $e_{3}=\rho$, where $i^{2}=-1$ and $\rho^{2}=0$.

P. W. Ketchum [26] considered the mentioned Segre algebra of quaternions as an example of harmonic algebra. Indeed, in this algebra the multiplication table for the basis $\{1, i, j, k\}$ is of the following form: $i^{2}=j^{2}=-1, \quad k^{2}=1, \quad i j=k, \quad i k=-j, \quad j k=-i$. Therefore, there are harmonic triads, in particular: $e_{1}=\sqrt{2}, e_{2}=i, e_{3}=j$.

At the same time, P. W. Ketchum [27] understood that it is impossible to obtain all solutions of Eq. (1.2) in the form of components of analytic functions taking values in a finite-dimensional commutative algebra. In the paper [27] he considered an infinite-dimensional vector space containing a harmonic triad. This vector space is not an algebra, but P. W. Ketchum proved that a set of components of analytic functions taking values in the mentioned space includes all analytic solutions of Eq. (1.2). M. N. Roşculeţ [79] considered an other infinite-dimensional vector space with a commutative multiplication for a part of its elements and functions generating solutions of Eq. (1.2).

L. Sobrero [97] considered a four-dimensional commutative associative algebra over the field $\mathbb{R}$ with the basis $\left\{1, j, j^{2}, j^{3}\right\}$ and the multiplication rule $j^{4}=-1-2 j^{2}$ that implies the equality $\left(1+j^{2}\right)^{2}=0$. Therefore, every analytic function $\Phi(\zeta)$ of the variable $\zeta=x+y j$ satisfies the biharmonic equation because

$$
\left(\frac{\partial^{4}}{\partial x^{4}}+2 \frac{\partial^{4}}{\partial x^{2} \partial y^{2}}+\frac{\partial^{4}}{\partial y^{4}}\right) \Phi(\zeta) \equiv \Phi^{(4)}(\zeta)\left(1+j^{2}\right)^{2}=0 .
$$


Note that the algebra considered in [97] is isomorphic to the mentioned algebra $\mathbb{B}$.

\section{Differentiable functions in commutative Banach algebras: theory and applications}

\subsection{Differentiability in the sense of Lorch and in the sense of Gâteaux}

It is clear that a characterization of functions satisfying the equalities (2.2) has relation to a question: in what sense the derivative is understood in the algebra $\mathbb{A}$.

It is well-known that there exist various definitions of differentiable functions given in algebras. Choosing a concept of differentiable function and its derivative, it is natural to desire to combine the largest set of functions satisfying the equalities (2.2) with the preservation of the basic properties of analytic functions of a complex variable for functions of the mentioned set.

Consider a function $\Phi: \Omega \longrightarrow \mathbb{A}$ given in a domain $\Omega \subset E_{3}$ and properties of differentiability of such a function.

The concepts of Fréchet derivative and Gâteaux derivative are used for mappings of linear normalized spaces. These derivatives are defined as linear operators. In the considered case, they are linear operators from $E_{3}$ into $\mathbb{A}$.

Formerly, for functions given in a domain of a finite-dimensional algebra, G. Scheffers [81] considered a derivative, which is understood as a function given in the same domain.

Generalizing such an approach to the case of mappings given in a domain of an arbitrary commutative associative Banach algebra, E.R. Lorch [37] introduced a derivative, which is also understood as a function given in the same domain.

A function $\Phi: \Omega \longrightarrow \mathbb{A}$ is called differentiable in the sense of Lorch (cf. [37]) in a domain $\Omega \subset E_{3}$ if for every $\zeta \in \Omega$ there exists an element $\Phi_{L}^{\prime}(\zeta) \in \mathbb{A}$ such that for any $\varepsilon>0$ there exists $\delta>0$ such that for all $h \in E_{3}$ with $\|h\|<\delta$ the following inequality fulfilled:

$$
\left\|\Phi(\zeta+h)-\Phi(\zeta)-h \Phi_{L}^{\prime}(\zeta)\right\| \leq\|h\| \varepsilon .
$$

Obviously, in the inequality (3.1) the Lorch derivative $\Phi_{L}^{\prime}(\zeta)$ is a function of the variable $\zeta$, i.e., $\Phi_{L}^{\prime}: \Omega \longrightarrow \mathbb{A}$.

At the same time, the mapping $B_{\zeta}: E_{3} \longrightarrow \mathbb{A}$, which is defined by the equality $B_{\zeta} h:=h \Phi_{L}^{\prime}(\zeta)$, is a bounded linear operator. Therefore, a function $\Phi$, which is differentiable in the sense of Lorch in a domain $\Omega$, 
have the Fréchet derivative $B_{\zeta}$ in every point $\zeta \in \Omega$ (cf. [25, p. 115]). The converse is not true, see an example in [25, p. 116].

Some properties similar to properties of analytic functions of a complex variable are established for functions differentiable in the sense of Lorch [37] in an arbitrary convex domain of commutative Banach algebra. In particular, the integral Cauchy theorem and the integral Cauchy formula, the Taylor expansion and the Morera theorem are proved in [37] in such a way as for analytic functions of a complex variable. The convexity of domain in the mentioned results from [37] is withdrawn by E. K. Blum [2].

Using the Gâteaux differential, I. P. Mel'nichenko [40] suggested to consider the Gâteaux derivative as a function $\Phi_{G}^{\prime}: \Omega \longrightarrow \mathbb{A}_{3}$ too.

We say that a function $\Phi: \Omega \longrightarrow \mathbb{A}$ is called differentiable in the sense of Gâteaux in a domain $\Omega \subset E_{3}$ if for every $\zeta \in \Omega$ there exists an element $\Phi_{G}^{\prime}(\zeta) \in \mathbb{A}$ such that

$$
\lim _{\delta \rightarrow 0+0}(\Phi(\zeta+\delta h)-\Phi(\zeta)) \delta^{-1}=h \Phi_{G}^{\prime}(\zeta) \quad \forall h \in E_{3}
$$

Obviously, the Gateaux derivative $\Phi_{G}^{\prime}(\zeta)$ is a function of the variable $\zeta$ and is a generalization of the classical directional derivative.

The left-hand side of equality (3.2) is called the Gâteaux differential of function $\Phi$. It is well-known, in a general case, the Gâteaux differential may fail to be linear with respect to $h$. But, it is clear, if the Gâteaux derivative $\Phi_{G}^{\prime}(\zeta)$ exists, the Gâteaux differential (3.2) is a bounded linear operator with respect to $h$. At the same time, the converse is not true as the same example in $[25$, p. 116] shows.

It is evident, both the definition (3.1) of the Lorch derivative and the definition (3.2) of the Gâteaux derivative take into account the existence of noninvertible elements $h$ in the algebra $\mathbb{A}$ because the division by elements of algebra is not used in them in contrast to the classical definition of complex derivative for functions of a complex variable.

Obviously, if a function $\Phi$ is differentiable in the sense of Lorch in $\Omega$, then it is also differentiable in the sense of Gâteaux, and $\Phi_{L}^{\prime}(\zeta)=\Phi_{G}^{\prime}(\zeta)$ for all $\zeta \in \Omega$. The converse is clearly not true similarly to the fact that the existence of all directional derivatives at a point does not guarantee a strong differentiability (or even continuity) of function at that point.

For a simplicity, let us consider triads $\left\{e_{1}, e_{2}, e_{3}\right\}$ containing unit of the algebra $\mathbb{A}$, i.e., for instance, let us set $e_{1}=1$. It follows evidently from equality (3.2) that if a function $\Phi: \Omega \longrightarrow \mathbb{A}$ is differentiable in the sense of Gâteaux in a domain $\Omega$, then there exist all partial derivatives 
$\partial \Phi / \partial x, \partial \Phi / \partial y, \partial \Phi / \partial z$ and the following conditions are satisfied in $\Omega$ :

$$
\frac{\partial \Phi}{\partial y}=\frac{\partial \Phi}{\partial x} e_{2}, \quad \frac{\partial \Phi}{\partial z}=\frac{\partial \Phi}{\partial x} e_{3} .
$$

The converse statement is not true as it is well-known in classical complex analysis because the existence of partial derivatives of a function of a complex variable together with Cauchy-Riemann conditions is not sufficient for the complex differentiability of this function.

In the case of finite-dimensional algebras the following statement is proved in a similar way as the analogous theorem on differentiability of functions of a complex variable: if the dimension n of algebra $\mathbb{A}$ is finite and in the expansion

$$
\Phi\left(x e_{1}+y e_{2}+z e_{3}\right)=\sum_{k=1}^{n} U_{k}(x, y, z) e_{k},
$$

of a function $\Phi: \Omega \longrightarrow \mathbb{A}$ with respect to the basis $\left\{e_{1}, e_{2}, \ldots, e_{n}\right\}$ of the algebra $\mathbb{A}$ the functions $U_{k}$ are $\mathbb{R}$-differentiable in $\Omega$, i.e.

$$
\begin{gathered}
U_{k}(x+\Delta x, y+\Delta y, z+\Delta z)-U_{k}(x, y, z) \\
=\frac{\partial U_{k}(x, y, z)}{\partial x} \Delta x+\frac{\partial U_{k}(x, y, z)}{\partial y} \Delta y+\frac{\partial U_{k}(x, y, z)}{\partial z} \Delta z \\
+o\left(\sqrt{(\Delta x)^{2}+(\Delta y)^{2}+(\Delta z)^{2}}\right), \quad(\Delta x)^{2}+(\Delta y)^{2}+(\Delta z)^{2} \rightarrow 0,
\end{gathered}
$$

and the conditions (3.3) are satisfied in $\Omega$, then the function $\Phi$ is differentiable in the sense of Lorch in $\Omega$.

\subsection{An algebraic-analytic approach to principal elliptic equations of mathematical physics}

I. P. Mel'nichenko [40] suggested to consider doubly differentiable in the sense of Gâteaux functions in the equalities (2.2).

Indeed, taking consistently basic elements $e_{1}, e_{2}, e_{3}$ as the vector $h$ in equalities of the form (3.2) which define the derivatives $\Phi_{G}^{\prime}(\zeta)$ and $\Phi_{G}^{\prime \prime}(\zeta)$, we obtain the equalities

$$
\frac{\partial^{2} \Phi}{\partial x^{2}}=e_{1}^{2} \Phi_{G}^{\prime \prime}(\zeta), \quad \frac{\partial^{2} \Phi}{\partial y^{2}}=e_{2}^{2} \Phi_{G}^{\prime \prime}(\zeta), \quad \frac{\partial^{2} \Phi}{\partial z^{2}}=e_{3}^{2} \Phi_{G}^{\prime \prime}(\zeta),
$$

Therefore, $\quad \Delta_{3} \Phi(\zeta) \equiv \Phi_{G}^{\prime \prime}(\zeta)\left(e_{1}^{2}+e_{2}^{2}+e_{3}^{2}\right), \quad \zeta=x e_{1}+y e_{2}+z e_{3}$.

Thus, if the basic elements $e_{1}, e_{2}, e_{3}$ satisfy the condition (2.1), then every doubly differentiable in the sense of Gâteaux function $\Phi: \Omega \longrightarrow \mathbb{A}$ 
satisfies Eq. (1.2) in $\Omega$. In turn, if there exists a doubly differentiable in the sense of Gâteaux function $\Phi: \Omega \longrightarrow \mathbb{A}$ satisfying Eq. (1.2) and the element $\Phi_{G}^{\prime \prime}(\zeta)$ is invertible at least at one point $\zeta \in \Omega$, then in this case the equality (2.1) holds.

I. P. Mel'nichenko [40] started to develop an algebraic-analytic approach to principal elliptic equations of mathematical physics. An idea of such an approach means a finding of commutative associative Banach algebra such that differentiable in the sense of Gâteaux functions with values in this algebra have components satisfying the given equations with partial derivatives.

Such algebras are found for the three-dimensional Laplace equation (see I. P. Mel'nichenko [40,42]), the two-dimensional biharmonic equation (see V. F. Kovalev and I. P. Mel'nichenko [30], I. P. Mel'nichenko [41]), the generalized biharmonic equation (see V. F. Kovalev and I. P. Mel'nichenko [31]) and elliptic equations degenerating on an axis that describe axialsymmetric potential fields (see I. P. Mel'nichenko and S. A. Plaksa [43]).

Note that differentiable in the sense of Gâteaux functions taking values in a commutative Banach algebra form a functional algebra, i.e. a very wide class of functions. Therefore, they can be simply constructed. Thus, a relation between these functions and solutions of equations with partial derivatives is important for constructing the mentioned solutions.

Let us note also that a priori the differentiability of a function in the sense of Gâteaux is a restriction being weaker than the differentiability of this function in the sense of Lorch.

In addition, differentiable in the sense of Lorch functions in commutative Banach algebras over the field $\mathbb{C}$ can be constructed in the form of principal extensions (see, e.g., [25, p. 165]) of analytic functions of a complex variable in particular. If a complex function $F$ is analytic in a domain $D \subset \mathbb{C}$, then for all $\zeta \in \mathbb{A}$ for which the spectrum is located in $D$, the principal extension of function $F$ can be expressed by the equality

$$
\frac{1}{2 \pi i} \int_{\Gamma_{\zeta}} F(t)(t-\zeta)^{-1} d t,
$$

where $\Gamma_{\zeta}$ is an arbitrary closed rectifiable Jordan curve in $D$ that embraces the spectrum of element $\zeta$.

\subsection{Three-dimensional harmonic algebras}

K. S. Kunz [34] developed a method for a formal construction of solutions of Eq. (1.2) with using power series in any harmonic algebra over the field $\mathbb{C}$. At the same time, he has emphasized that there does not appear to be a suitable three-dimensional harmonic algebra. 
The problem on finding a three-dimensional harmonic algebra $\mathbb{A}$ with unit was completely solved by I. P. Mel'nichenko $[40,42,45]$. In the paper [40] I. P. Mel'nichenko established that there does not exist harmonic bases $\left\{e_{1}, e_{2}, e_{3}\right\}$ with $e_{1}=1$ in three-dimensional commutative algebras over the field $\mathbb{R}$, but he constructed a three-dimensional harmonic algebra over the field $\mathbb{C}$.

Theorem 3.1 (I. P. Mel'nichenko [40]). The commutative associative algebra $\mathbb{A}$ is harmonic, if the multiplication table for the basis $\left\{e_{1}, e_{2}, e_{3}\right\}$ is of the following form:

$$
\begin{aligned}
e_{k} e_{1} & =e_{k}, \quad k=1,2,3 \\
e_{2} e_{2} & =-\frac{1}{2} e_{1}-\frac{i}{2}(\sin \omega) e_{2}+\frac{i}{2}(\cos \omega) e_{3}, \\
e_{2} e_{3} & =\frac{i}{2}(\cos \omega) e_{2}+\frac{i}{2}(\sin \omega) e_{3}, \\
e_{3} e_{3} & =-\frac{1}{2} e_{1}+\frac{i}{2}(\sin \omega) e_{2}-\frac{i}{2}(\cos \omega) e_{3},
\end{aligned}
$$

where $i$ is the imaginary complex unit and $\omega \in \mathbb{C}$.

If a function $\Phi: \Omega \rightarrow \mathbb{A}$ is differentiable in the sense of Gâteaux in a domain $\Omega \subset E_{3}$, then the components $U_{k}, k=1,2,3$, of expansion (3.4) with $n=3$ generate the vectors $\mathbf{V}_{\mathbf{1}}:=\left(\operatorname{Re} U_{1},-\frac{1}{2} \operatorname{Re} U_{2},-\frac{1}{2} \operatorname{Re} U_{3}\right)$, $\mathbf{V}_{\mathbf{2}}:=\left(\operatorname{Im} U_{1},-\frac{1}{2} \operatorname{Im} U_{2},-\frac{1}{2} \operatorname{Im} U_{3}\right)$ such that their coordinates satisfy Eqs. (1.1).

To prove Theorem 3.1 I. P. Mel'nichenko as well as H. A. v. BeckhWidmanstetter [1] wrote out a system of algebraic equations for structured constants of algebra and showed that this system has only complex solutions.

Later I. P. Mel'nichenko [42] has developed a more simple method of the proof which is based on the description of all commutative associative algebras of a certain dimension, and the problem on finding a harmonic algebra is concretized as the problem on finding harmonic bases in concrete algebras. As a result, I. P. Mel'nichenko [42,45] found all three-dimensional harmonic algebras and developed a method for finding all harmonic bases in these algebras.

Theorem 3.2 (I. P. Mel'nichenko [40]). There does not exist any harmonic basis in a three-dimensional commutative algebra with unit over the field $\mathbb{R}$.

Theorem 3.3 (I. Mel'nichenko $[42,45])$. The algebra $\mathbb{A}_{4}$ is not harmonic. The algebras $\mathbb{A}_{1}, \mathbb{A}_{2}, \mathbb{A}_{3}$ are harmonic. 
All harmonic bases in the algebras $\mathbb{A}_{1}, \mathbb{A}_{2}, \mathbb{A}_{3}$ are described (see [45]). Note that in the semisimple algebra $\mathbb{A}_{1}$, in particular, there are the family of harmonic bases constructed in Theorem 3.1.

\subsection{Monogenic functions}

Let us consider a concept of monogenic function $\Phi: \Omega \longrightarrow \mathbb{A}$.

We say that a function $\Phi: \Omega \longrightarrow \mathbb{A}$ is monogenic in a domain $\Omega \subset E_{3}$ if $\Phi$ is continuous and differentiable in the sense of Gâteaux at every point of $\Omega$.

We use the notion of monogenic function in the sense of existence of derived numbers for this function (cf. [21,101]) in a combination to its continuity.

In the scientific literature the denomination of monogenic function is used else for functions given in non-commutative algebras and satisfying certain conditions similar to the classical Cauchy-Riemann conditions (see, e.g., [80]). The latter functions are also called regular functions (see, e.g., [99]) or hyperholomorphic functions (see, e.g., [32]).

In the papers $[55,59-62,65,70,75,76,83,94,95]$, we considered monogenic functions in harmonic algebras to prove for these functions analogues of principal theorems of the analytic function theory in the complex plane. We developed the following research scheme:

- at first, it is useful to obtain a constructive description of monogenic functions by means of analytic functions of complex variables;

- hereupon, to show that monogenic functions have the continuous Gâteaux derivatives of all orders and are differentiable in the sense of Lorch as well;

- at last, to prove integral theorems and to obtain the Taylor and Laurent expansions.

Some component parts of the mentioned research scheme are developed by I. P. Mel'nichenko and S. A. Plaksa (see [43, 45, 55]) for monogenic functions in an infinite-dimensional algebra associated with axial symmetric potential fields and by I. P. Mel'nichenko and V. F. Kovalev (see $[29,30])$, S. V. Grishchuk and S. A. Plaksa (see $[12,14,55,59]$ ) for monogenic functions in the algebra associated with the biharmonic equation. V. S. Shpakivskyi [84-86] has extended such a scheme to the case of monogenic functions in an arbitrary finite-dimensional commutative associative algebra.

Let us to outline a way to get constructive descriptions of monogenic functions by means of analytic functions of complex variables. 
In the theory of commutative Banach algebras there is the following fundamental fact (see, e.g., [25, p. 145]): for any commutative Banach algebra $\mathbb{A}$ with unit over the complex field $\mathbb{C}$ and for any maximal ideal $\mathfrak{I}$ of the algebra $\mathbb{A}$, the quotient algebra $\mathbb{A} / \mathfrak{I}$ is isomorphic to $\mathbb{C}$.

Let $f: \mathbb{A} \longrightarrow \mathbb{C}$ be the linear multiplicative functional such that $\mathfrak{I}$ is its kernel and $f(1)=1$ (see, e.g., [25, p. 146]).

The initial point of the mentioned way is the following statement: if for a monogenic function $\Phi: \Omega \longrightarrow \mathbb{A}$ the domain $\Omega$ is convex "in the direction" of a maximal ideal $\mathfrak{I}$ and $\zeta_{1}-\zeta_{2} \in \mathfrak{I}$, then the difference $\Phi\left(\zeta_{1}\right)-\Phi\left(\zeta_{2}\right)$ belongs to to the same ideal.

Hereupon, we can define the linear operator $A$ which assigns the function $F: D \longrightarrow \mathbb{C}$ to a monogenic function $\Phi: \Omega \longrightarrow \mathbb{A}$ by the formula $F(f(\zeta)):=f(\Phi(\zeta))$ for all $\zeta \in \Omega$. The function $F$ is given in the domain $D:=f(\Omega)$, where $f(\Omega)$ is the image of $\Omega$ under the mapping $f$. Moreover, $F$ is an analytic function in $D$ due to Ju.Ju. Trokhimchuk Theorem 21 in [101].

Considering a generalized inverse operator $A^{(-1)}$ which satisfies the equality $A A^{(-1)} A=A$, one can make sure that values of the monogenic function $\Phi-A^{(-1)} A \Phi$ belong to the ideal $\mathfrak{I}$ for every monogenic function $\Phi: \Omega \longrightarrow \mathbb{A}$.

Finally, in the case of an arbitrary finite-dimensional commutative associative algebra $\mathbb{A}$, it is possible to integrate the conditions (3.3) for a monogenic function $\Phi: \Omega \longrightarrow \mathfrak{I}$ and to describe all monogenic functions taking values in the ideal $\mathfrak{I}$ by means of analytic functions of complex variables due to G. Tolstoff result in [100].

\subsection{Monogenic functions in three-dimensional harmonic algebras}

For the first time, the mentioned research scheme was realized in the papers $[55,59,70,83,94,95]$ for monogenic functions in a three-dimensional harmonic algebra $\mathbb{A}_{3}$ with two-dimensional radical.

Let $\mathbb{A}=\mathbb{A}_{3}$, and, for a simplicity, consider a concrete harmonic basis in $\mathbb{A}_{3}$, viz., $e_{1}=1, e_{2}=i+\rho_{2}, e_{3}=(1-i) \rho_{1}$. In this case, all noninvertible elements in $E_{3}$ are located on the axis $O z$ and belong to the radical which is the unique maximal ideal in the algebra $\mathbb{A}_{3}$.

We say that a domain $\Omega \subset \mathbb{R}^{3}$ is convex in the direction of straight line $L$ if $\Omega$ contains each segment that connects two points $\left(x_{1}, y_{1}, z_{1}\right)$, $\left(x_{2}, y_{2}, z_{2}\right) \in \Omega$ and is parallel to $L$.

A constructive description of monogenic functions taking values in the algebra $\mathbb{A}_{3}$ by means of analytic functions of a complex variable is obtained in the paper [70]: 
Theorem 3.4. If a domain $\Omega$ is convex in the direction of axis $O z$, then for any monogenic function $\Phi: \Omega \longrightarrow \mathbb{A}_{3}$ there exists such a triad of complex functions $F, F_{1}, F_{2}$ analytic in the domain $D=\{x+i y$ : $\left.x e_{1}+y e_{2}+z e_{3} \in \Omega\right\}$ that $\Phi$ can be expressed in the form:

$$
\begin{aligned}
\Phi(\zeta)= & F(\xi)+\left((1-i) z F^{\prime}(\xi)+F_{1}(\xi)\right) \rho_{1} \\
& +\left(y F^{\prime}(\xi)-i z^{2} F^{\prime \prime}(\xi)+(1-i) z F_{1}^{\prime}(\xi)+F_{2}(\xi)\right) \rho_{2}, \\
& \xi=x+i y, \quad \forall \zeta=x e_{1}+y e_{2}+z e_{3} \in \Omega .
\end{aligned}
$$

Note that the condition of convexity of $\Omega$ in the direction of axis $O z$ is essential for the truth of Theorem 3.4 as it is shown by means of construction of an example (see [55, 59,70]).

Equality (3.6) can be rewritten in the following form (see [55])

$$
\Phi(\zeta)=\frac{1}{2 \pi i} \int_{\Gamma_{\zeta}}\left(F(t)+\rho_{1} F_{1}(t)+\rho_{2} F_{2}(t)\right)(t-\zeta)^{-1} d t \quad \forall \zeta \in \Omega,
$$

where $\Gamma_{\zeta}$ is an arbitrary closed Jordan rectifiable curve in $D$, which is homotopic to the point $\xi$ and embraces this point, i.e. $\Phi$ is expressed via the principal extensions of analytic functions $F, F_{1}, F_{2}$ into the domain $\Omega$.

It follows from equality (3.7) that the function $\Phi$ is differentiable in the sense of Lorch in $\Omega$. Using equality (3.7), we obtain the following expression for the Lorch $n$-th derivative, which coincides with the Gateaux $n$-th derivative:

$$
\Phi^{(n)}(\zeta)=\frac{n !}{2 \pi i} \int_{\Gamma_{\zeta}}\left(F(t)+\rho_{1} F_{1}(t)+\rho_{2} F_{2}(t)\right)\left((t-\zeta)^{-1}\right)^{n+1} d t \quad \forall \zeta \in \Omega .
$$

Let us note that in the case of monogenic function $\Phi: \Omega \longrightarrow \mathbb{A}_{3}$ given in an arbitrary domain $\Omega$, equalities (3.6)-(3.8) are fulfilled at least locally, i.e., in a neighborhood of each point $\zeta \in \Omega$.

Thus, every monogenic function $\Phi: \Omega \longrightarrow \mathbb{A}_{3}$ satisfies equalities $(2.2)$ in $\Omega$. Moreover, it follows from equality (3.6) that its components $U_{k}$, $k=1,2,3$, of expansion (3.4) with $n=3$ are $\mathbb{R}$-differentiable in $\Omega$.

In the papers $[55,83,94,95]$, for monogenic functions $\Phi: \Omega \longrightarrow \mathbb{A}_{3}$ given in an arbitrary domain $\Omega$, we established basic properties analogous to properties of analytic functions of a complex variable: the Cauchy integral theorem and integral formula, the Morera theorem, the uniqueness theorem, the Taylor expansions. 
In contrast to similar results in the papers E. R. Lorch [37] and E. K. Blum [2], monogenic functions $\Phi: \Omega \longrightarrow \mathbb{A}_{3}$ are given only in a domain $\Omega$ of the linear span $E_{3}$ instead of domain of whole algebra.

The proof of the following statement comprising the integral Cauchy formula for monogenic functions $\Phi: \Omega \longrightarrow \mathbb{A}_{3}$ can be found in the papers $[55,94,95]$ :

Theorem 3.5. Let $\Omega$ be a domain convex in the direction of axis $O z$ and $\Phi: \Omega \longrightarrow \mathbb{A}_{3}$ be a monogenic function in $\Omega$. Then for every point $\zeta_{0} \in \Omega$ the following equality is true:

$$
\Phi\left(\zeta_{0}\right)=\frac{1}{2 \pi i} \int_{\gamma_{\zeta}} \Phi(\zeta)\left(\zeta-\zeta_{0}\right)^{-1} d \zeta,
$$

where $\gamma_{\zeta}$ is an arbitrary closed Jordan rectifiable curve in $\Omega$, which around once the straight line $\left\{\zeta_{0}+z e_{3}: z \in \mathbb{R}\right\}$ and is homotopic to the point $\zeta_{0}$.

Note that the integral Cauchy formula established in the papers $[2,37]$ is not applicable to a monogenic function $\Phi: \Omega \longrightarrow \mathbb{A}_{3}$ because it deals with an integration along a curve on which the function $\Phi$ is not given, generally speaking.

The following theorem giving different equivalent definitions of monogenic functions is true (cf. [55,95]):

Theorem 3.6. A function $\Phi: \Omega \rightarrow \mathbb{A}_{3}$ is monogenic in an arbitrary domain $\Omega$ if and only if one of the following conditions is satisfied:

(I) the components $U_{k}, k=1,2,3$, of expansion (3.4) with $n=3$ are $\mathbb{R}$-differentiable and the conditions (3.3) are satisfied in the domain $\Omega$;

(II) in every ball $\mho \subset \Omega$ the function $\Phi$ is expressed in the form (3.6), where the triad of analytic in the domain $f(\mho)$ functions $F, F_{1}, F_{2}$ is unique;

(III) the function $\Phi$ is continuous in $\Omega$ and satisfies the equality

$$
\int_{\partial \triangle} \Phi(\zeta) d \zeta=0
$$

for every triangle $\triangle \subset \Omega$ that is understood as a plane figure bounded by three line segments connecting three its vertices, and $\partial \triangle$ is the boundary of triangle $\triangle$ in relative topology of its plane;

(IV) the function $\Phi$ is analytic in $\Omega$, i.e. for every $\zeta_{0} \in \Omega$ there exists a neighborhood, in which the function $\Phi$ is expressed as the sum of power series

$$
\Phi(\zeta)=\sum_{k=0}^{\infty} c_{k}\left(\zeta-\zeta_{0}\right)^{k}, \quad c_{k} \in \mathbb{A}_{3} .
$$


In the paper [57], we show that any function $\Phi: \Omega \longrightarrow \mathbb{A}_{3}$ which are differentiable in the sense of Gâteaux and locally bounded in $\Omega$ is also monogenic in $\Omega$.

Let us write down constructive descriptions of monogenic functions taking values in either the algebra $\mathbb{A}_{1}$ or the algebra $\mathbb{A}_{2}$.

For a simplicity, consider a concrete harmonic basis in $\mathbb{A}_{2}$, viz., $e_{1}=1$, $e_{2}=i \mathcal{I}_{1}+\rho, e_{3}=i \mathcal{I}_{2}$. In this case, all noninvertible elements in $E_{3}$ are located on the axes $O y$ and $O z$.

Consider also a concrete harmonic basis in $\mathbb{A}_{1}$, viz., $e_{1}=1, e_{2}=i \mathcal{I}_{1}+$ $\frac{\sqrt{2}}{2} i \mathcal{I}_{3}, e_{3}=i \mathcal{I}_{2}-\frac{\sqrt{2}}{2} i \mathcal{I}_{3}$. In this case, all noninvertible elements in $E_{3}$ are located on the axes $O y, O z$ and on the straight line $L:=\left\{t\left(e_{2}+e_{3}\right)\right.$ : $t \in \mathbb{R}\}$.

Denote $D_{1}:=\left\{x+i y: x e_{1}+y e_{2}+z e_{3} \in \Omega\right\}, D_{2}:=\{x+i z:$ $\left.x e_{1}+y e_{2}+z e_{3} \in \Omega\right\}, D_{3}:=\left\{x+\frac{\sqrt{2}}{2} i(y-z): x e_{1}+y e_{2}+z e_{3} \in \Omega\right\}$.

Now, we can formulate the following theorems similar to Theorem 3.4:

Theorem 3.7 ([76]). Let $\mathbb{A}=\mathbb{A}_{1}$ and a domain $\Omega \subset E_{3}$ be convex in the directions of axes $\mathrm{Oy}, \mathrm{Oz}$ and straight line $L$. Then every monogenic function $\Phi: \Omega \longrightarrow \mathbb{A}_{1}$ can be expressed in the form

$$
\Phi(\zeta)=F_{1}\left(\xi_{1}\right) \mathcal{I}_{1}+F_{2}\left(\xi_{2}\right) \mathcal{I}_{2}+F_{3}\left(\xi_{3}\right) \mathcal{I}_{3} \quad \forall \zeta=x e_{1}+y e_{2}+z e_{3} \in \Omega,
$$

where $F_{k}$ is an analytic function in the domain $D_{k}$ for $k=1,2,3$ and $\xi_{1}=x+i y, \xi_{2}=x+i z, \xi_{3}=x+\frac{\sqrt{2}}{2} i(y-z)$.

Theorem 3.8 ([60]). Let $\mathbb{A}=\mathbb{A}_{2}$ and a domain $\Omega \subset E_{3}$ be convex in the directions of axes $O y$ and $O z$. Then every monogenic function $\Phi: \Omega \longrightarrow \mathbb{A}_{2}$ can be expressed in the form

$$
\begin{aligned}
\Phi(\zeta)=F_{1}\left(\xi_{1}\right) \mathcal{I}_{1} & +F_{2}\left(\xi_{2}\right) \mathcal{I}_{2} \\
& +\left(y F_{2}^{\prime}\left(\xi_{2}\right)+F_{0}\left(\xi_{2}\right)\right) \rho \quad \forall \zeta=x e_{1}+y e_{2}+z e_{3} \in \Omega,
\end{aligned}
$$

where $F_{1}$ is an analytic function in the domain $D_{1}$ and $F_{2}, F_{0}$ are analytic functions in the domain $D_{2}$, and $\xi_{1}=x+i y, \xi_{2}=x+i z$.

The mentioned way to get constructive descriptions of monogenic functions by means of analytic functions of complex variables in Theorems 3.7 and 3.8 is implemented. At the same time, let us note that on such a way it is impossible to use the principal extensions of analytic functions of a complex variable just as it was made earlier in the papers $[12,55,59,70]$ for monogenic functions taking values in algebras which contain the unique maximal ideal. Nevertheless, it is possible to 
construct in explicit form operators which assign monogenic functions in the given domain $\Omega$ to complex analytic functions (see $[60,61,76]$ ).

Theorems 3.4, 3.7 and 3.8 are basic for the proof of analogues of the classical Gauss-Ostrogradskii and Stokes formulas as well as Cauchy integral theorems for both surface and curvilinear integrals. Such theorems are proved in the papers $[55,94,95]$ for functions taking values in the algebra $\mathbb{A}_{3}$, but, as it follows from their proof, they are true for monogenic functions in any three-dimensional harmonic algebra.

The Laurent expansions of monogenic functions taking values in the algebra $\mathbb{A}_{3}$ are obtained and singularities of these functions are classified by V. S. Shpakivskyi [83]. In particular, it is shown that an isolated singular point of monogenic function can be only removable. In the case where a function has a unremovable singularity at the point $\zeta_{0}$, all points of the line $\left\{\zeta_{0}+z e_{3}: z \in \mathbb{R}\right\}$ are singular. The Taylor and Laurent expansions of monogenic functions taking values in the algebra $\mathbb{A}_{2}$ are obtained by R. P. Pukhtaievych [75].

We computed the logarithmic residue for monogenic functions taking values in either the algebra $\mathbb{A}_{3}$ in [71] or the algebra $\mathbb{A}_{2}$ in [77]. It is shown that the logarithmic residue depends not only on zero points and singular points of a function but also on the points at which the function takes values in the ideals of algebra. Some earlier similar result was obtained in [13] for monogenic functions taking values in the two-dimensional algebra $\mathbb{B}$ mentioned above.

In the papers $[63,64]$ we established sufficient conditions for the existence of limiting values of an analog of the Cauchy type integral taking values in the algebra $\mathbb{A}_{3}$.

\subsection{Monogenic functions in finite-dimensional commutative associative algebras}

Using the multiplication table proposed by E. Cartan [4] for a basis in any finite-dimensional associative algebra with unit, V. S. Shpakivskyi [84-86] has developed a scheme of research to the case of monogenic functions taking values in finite-dimensional commutative algebras. In the papers $[84,85]$ he obtained constructive descriptions of monogenic functions taking values in an arbitrary finite-dimensional commutative algebra by means of analytic functions of a complex variable. These result generalizes Theorems 3.4, 3.7 and 3.8 as well as corresponding results from the papers $[62,65]$ and summarizes all results on constructive descriptions of analytic functions in concrete finite-dimensional commutative algebras that were begun by F. Ringleb [78], who obtained such a description for analytic functions of a bicomplex variable. For the 
mentioned monogenic functions V. S. Shpakivskyi [86] proved curvilinear analogues of the Cauchy integral theorem, the Morera theorem and the Cauchy integral formula.

V. S. Shpakivskyi and T. S. Kuzmenko [35,91-93] used the mentioned research scheme in a noncommutative algebra of complex quaternions. They defined classes of quaternion mappings having properties similar to properties of monogenic functions in commutative algebras.

In [67], we proved an analogue of the Cauchy integral theorem for a surface integral of hyperholomorphic functions given in three-dimensional domains with non piece-smooth boundaries and taking values in an arbitrary finite-dimensional commutative associative Banach algebra. Note that monogenic functions in harmonic algebras form a subset of hyperholomorphic functions considered in [67]. Similar analogue of the Cauchy integral theorem is proved by O. F. Herus [24] for hyperholomorphic functions given in a noncommutative algebra of quaternions.

Studying characteristic properties of monogenic functions facilitates to developing hypercomplex methods for solving problems of mathematical physics. V. S. Shpakivskyi [87-90] has shown that for the construction of solutions of linear PDEs with constant coefficients in the form of components of monogenic functions with values in finite-dimensional commutative associative algebras, it is sufficient to use monogenic functions in algebras of a special form, where all idempotents are substituted by unit. He proposed a procedure for the construction of an infinite family of solutions by means of monogenic functions given in a sequence of concrete algebras. The proposed method is used to construct solutions of some equations of mathematical physics.

Some examples of the application of differentiable in the sense of Gâteau functions to PDEs with several variables are considered by A. Pogorui, R. M. Rodriguez-Dagnino and M. Shapiro [72].

An interest in the study of functions given in commutative algebras of hypercomplex numbers is increased in recent years due to advantages of commutativity together with possibilities of various applications. Some algebraic, geometric and analytic aspects of theory of hypercomplex numbers are studied in monographs of G. B. Price [74], D. Boccaletti et al. [3], M. E. Luna-Elizarrarás et al. [38]. V. V. Kisil [28] proposed to classify different analytic function theories in relation to underlying symmetries.

\subsection{Monogenic functions in a biharmonic algebra and BVPs for biharmonic functions}

An associative commutative two-dimensional algebra with unit over the complex field is called biharmonic (see V. F. Kovalev and I. P. Mel'ni- 
chenko [30]) if in this algebra there exists a biharmonic basis $\left\{e_{1}, e_{2}\right\}$ satisfying the conditions

$$
\left(e_{1}^{2}+e_{2}^{2}\right)^{2}=0, \quad e_{1}^{2}+e_{2}^{2} \neq 0 .
$$

V. F. Kovalev and I. P. Mel'nichenko [30] found a multiplication table for a biharmonic basis $\left\{e_{1}, e_{2}\right\}: \quad e_{1}=1, \quad e_{2}^{2}=e_{1}+2 i e_{2}$.

I. P. Mel'nichenko [41] proved that there exists the unique biharmonic algebra $\mathbb{B}$ mentioned above, and he found all biharmonic bases in $\mathbb{B}$. Note that the algebra $\mathbb{B}$ is isomorphic to four-dimensional over the real field algebras considered by L. Sobrero [97] and A. Douglis [6].

Consider a biharmonic plane $\mu_{e_{1}, e_{2}}:=\left\{\zeta=x e_{1}+y e_{2}: x, y \in \mathbb{R}\right\}$. Let $G$ be a domain in the plane $\mu_{e_{1}, e_{2}}$. Inasmuch as divisors of zero do not belong to the plane $\mu_{e_{1}, e_{2}}$, the Gâteaux derivative of function $\Phi: G \longrightarrow \mathbb{B}$ coincides with the derivative

$$
\Phi^{\prime}(\zeta):=\lim _{h \rightarrow 0, h \in \mu_{e_{1}, e_{2}}}(\Phi(\zeta+h)-\Phi(\zeta)) h^{-1} .
$$

Therefore, a function $\Phi: G \longrightarrow \mathbb{B}$ is monogenic in a domain $G$ if the derivative $\Phi^{\prime}(\zeta)$ exists at every point $\zeta \in G$.

Let us use the same denotation $G$ for a domain $G \subset \mathbb{R}^{2}$ and for the domains in $\mu_{e_{1}, e_{2}}$ and $\mathbb{C}$ that are congruent to the domain $G$.

Any function $\Phi: G \longrightarrow \mathbb{B}$ has an expansion

$$
\Phi(\zeta)=U_{1}(x, y) e_{1}+U_{2}(x, y) i e_{1}+U_{3}(x, y) e_{2}+U_{4}(x, y) i e_{2},
$$

where $U_{1}, U_{2}, U_{3}, U_{4}$ are real-valued functions.

V. F. Kovalev and I. P. Mel'nichenko [30] established that a function $\Phi: G \longrightarrow \mathbb{B}$ is monogenic in a domain $G$ if and only if the components $U_{l}: G \longrightarrow \mathbb{R}, l=1,2,3,4$, in expansion (3.10) are $\mathbb{R}$-differentiable in $G$ and the following Cauchy-Riemann condition is satisfied:

$$
\frac{\partial \Phi(\zeta)}{\partial y}=\frac{\partial \Phi(\zeta)}{\partial x} e_{2}
$$

Furthermore, for principal extension (3.5) of analytic function $F$ : $G \longrightarrow \mathbb{C}$ into the congruent domain of biharmonic plane $\mu_{e_{1}, e_{2}}$ an expansion with respect to the biharmonic basis $\left\{e_{1}, e_{2}\right\}$ is obtained by V. F. Kovalev and I. P. Mel'nichenko [30].

Moreover, every monogenic function $\Phi: G \longrightarrow \mathbb{B}$ is expressed via two corresponding analytic functions $F: G \longrightarrow \mathbb{C}, F_{0}: G \longrightarrow \mathbb{C}$ of the complex variable $z=x+i y$ in the form (cf. $[12,55,59]$ ):

$$
\Phi(\zeta)=F(z) e_{1}-\left(\frac{i y}{2} F^{\prime}(z)-F_{0}(z)\right) \rho \quad \forall \zeta=x e_{1}+y e_{2} \in G .
$$


V. F. Kovalev [29] obtained equality (3.11) under additional assumptions on the geometry of domain $G$. The equality (3.11) establishes one-to-one correspondence between monogenic functions $\Phi$ and pairs of complexvalued analytic functions $F, F_{0}$.

Therefore, every monogenic function $\Phi: G \longrightarrow \mathbb{B}$ has the derivative of any order in $G$ (cf. $[12,55,59]$ ) and satisfies the equalities

$$
\left(\frac{\partial^{4}}{\partial x^{4}}+2 \frac{\partial^{4}}{\partial x^{2} \partial y^{2}}+\frac{\partial^{4}}{\partial y^{4}}\right) \Phi(\zeta)=\Phi^{(4)}(\zeta)\left(e_{1}^{2}+e_{2}^{2}\right)^{2}=0
$$

for all $\zeta=x e_{1}+y e_{2} \in G$ due to conditions (3.9).

Now, one can state that all components $U_{l}, l=1,2,3,4$, in expansion (3.10) of any monogenic function $\Phi: G \longrightarrow \mathbb{B}$ are biharmonic functions (cf. $[12,55,59])$, i.e., satisfy the biharmonic equation in $G$ :

$$
\frac{\partial^{4} U(x, y)}{\partial x^{4}}+2 \frac{\partial^{4} U(x, y)}{\partial x^{2} \partial y^{2}}+\frac{\partial^{4} U(x, y)}{\partial y^{4}}=0 .
$$

At the same time, every biharmonic in a simply connected domain $G$ function $U(x, y)$ is the first component $U_{1} \equiv U$ in expression (3.10) of a certain function $\Phi: G \longrightarrow \mathbb{B}$ monogenic in $G$ and, moreover, all such functions $\Phi$ are found in an explicit form (see $[12,55,59]$ ).

S. V. Gryshchuk [10] established similar relations between solutions of the generalized biharmonic equation and monogenic functions taking values in the semisimple algebra $\mathbb{B}_{0}$ mentioned above.

In the papers $[14,55]$, for monogenic functions taking values in the algebra $\mathbb{B}$, we established basic properties analogous to properties of analytic functions of a complex variable: the Cauchy integral theorem and integral formula, the Morera theorem, the uniqueness theorem, the Taylor and Laurent expansions. Thus, we proved a theorem (see $[14,55]$ ) which is similar to Theorem 3.6 and gives different equivalent definitions of monogenic functions in the biharmonic plane.

It is well-known that a technique of using of analytic functions of a complex variable for solving biharmonic BVPs is based on an expression of biharmonic functions by the Goursat formula (cf., e.g., [48,96]). This expression allows to reduce the biharmonic problems to certain BVPs for analytic functions. Further, expressing analytic functions via the Cauchy type integrals, one can obtain a system of integro-differential equations in the general case. In the case where the boundary is a Lyapunov curve, the mentioned system is reduced to a system of Fredholm equations. Such a scheme is developed for solving BVPs of the plane elasticity theory with using a special biharmonic function which is called the Airy stress function (cf., e.g., $[46,48])$. 
To develop new methods for effective solving BVPs of the plane elasticity theory, one can use relations of biharmonic functions with monogenic functions in the algebra $\mathbb{B}$.

Consider the following boundary value problem: to find a monogenic function $\Phi: G \longrightarrow \mathbb{B}$ when limiting values of two components of expansion (3.10) are given on the boundary $\partial G$, i.e. the following boundary conditions are satisfied:

$$
U_{k}(x, y)=u_{k}(x, y), \quad U_{m}(x, y)=u_{m}(x, y) \quad \forall(x, y) \in \partial G
$$

for $1 \leq k<m \leq 4$, where $u_{k}$ and $u_{m}$ are given functions.

This problem was posed by V. F. Kovalev [29]. He named such a problem as biharmonic Schwarz problem due to its analogy with the classical Schwarz problem on finding an analytic function of a complex variable when values of its real part are given on the boundary of domain.

We call such a problem by $(k-m)$-problem. V. F. Kovalev [29] established that all $(k-m)$-problems are reduced to the main three problems: (1-2)-problem or (1-3)-problem or (1-4)-problem.

V. F. Kovalev [29] shown that the main biharmonic problem (cf., e.g., $[96$, p. 194] and [46, p. 13]) on finding a biharmonic function $U: G \longrightarrow \mathbb{R}$ with given limiting values of its partial derivatives $\partial U / \partial x$ and $\partial U / \partial y$ on the boundary $\partial G$ can be reduced to the (1-3)-problem (see also $[15,16]$ ).

S. V. Gryshchuk [8] investigated a relation between (1-4)-problem and BVPs of the plane elasticity theory. He considered a problem on finding an elastic equilibrium for isotropic body occupying $G$ with given limiting values of partial derivatives $\partial u / \partial x, \partial v / \partial y$ for displacements $u=u(x, y)$, $v=v(x, y)$ on the boundary $\partial G$. It is shown in [8] that such a problem is reduced to (1-4)-problem (see also $[18,19]$ ).

V. F. Kovalev [29] solved (1-4)-problem for a half-plane in an explicit form under some natural conditions on the given functions. Further, using equality (3.11) and a conformal mapping of a domain $G$ onto a half-plane and (1-4)-problem as an auxiliary problem, he reduced (1-2)-problem and (1-3)-problem to integro-differential equations. Note that V. F. Kovalev [29] stated only a sketch of solving of biharmonic Schwarz problems and did not investigate conditions of solvability of these problems.

In the papers $[8,9,15-19]$, for solving biharmonic Schwarz problems, we developed methods which are based on expressions of solutions by hypercomplex integrals analogous to the classical Schwarz and Cauchy type integrals.

In [15], we investigated (1-3)-problem for the case where $G$ is either a half-plane or a unit disk in the biharmonic plane and found solutions in explicit forms with using of some integrals analogous to the classical 
Schwarz integral. In $[9,16]$, using a hypercomplex analog of the Cauchy type integral, we reduced the (1-3)-problem to a system of integral equations and established sufficient conditions under which this system has the Fredholm property. It was made for the case where the boundary of domain belongs to a class being wider than the class of Lyapunov curves that was usually required in the plane elasticity theory (cf., e.g., [46, 48]). The similar is done for the (1-4)-problem in $[17,18]$. Obtained results are mostly analogous to appropriate results in $[15,16]$ dealing with the (1-3)problem, but in contradistinction to the (1-3)-problem, which is solvable in a general case if and only if a certain natural condition is satisfied, the (1-4)-problem is solvable unconditionally.

\section{Monogenic functions in infinite-dimensional vector spaces with commutative multiplication}

\subsection{Infinite-dimensional vector spaces associated with the three-dimensional Laplace equation}

It is impossible to obtain all solutions of Eq. (1.2) in the form of components of monogenic functions taking values in a finite-dimensional commutative algebra (see, e.g., $[27,34,45,55])$. In particular, for such an algebra there exist spherical functions which are not components of specified hypercomplex monogenic functions.

Consider an infinite-dimensional commutative associative Banach algebra $\mathbb{F}:=\left\{g=\sum_{k=1}^{\infty} c_{k} e_{k}: c_{k} \in \mathbb{R}, \sum_{k=1}^{\infty}\left|c_{k}\right|<\infty\right\}$ over the field $\mathbb{R}$ with the norm $\|g\|_{\mathbb{F}}:=\sum_{k=1}^{\infty}\left|c_{k}\right|$ and the basis $\left\{e_{k}\right\}_{k=1}^{\infty}$, where the multiplication table for elements of basis is of the following form (cf. [45, 55]):

$$
\begin{gathered}
e_{n} e_{1}=e_{n}, \quad e_{2 n+1} e_{2 n}=\frac{1}{2} e_{4 n} \quad \forall n \geq 1, \\
e_{2 n+1} e_{2 m}=\frac{1}{2}\left(e_{2 n+2 m}-(-1)^{m} e_{2 n-2 m}\right) \quad \forall n>m \geq 1, \\
e_{2 n+1} e_{2 m}=\frac{1}{2}\left(e_{2 n+2 m}+(-1)^{n} e_{2 m-2 n}\right) \quad \forall m>n \geq 1, \\
e_{2 n+1} e_{2 m+1}=\frac{1}{2}\left(e_{2 n+2 m+1}+(-1)^{m} e_{2 n-2 m+1}\right) \quad \forall n \geq m \geq 1, \\
e_{2 n} e_{2 m}=\frac{1}{2}\left(-e_{2 n+2 m+1}+(-1)^{m} e_{2 n-2 m+1}\right) \quad \forall n \geq m \geq 1 .
\end{gathered}
$$

It is evident that $e_{1}, e_{2}, e_{3}$ form a harmonic triad of vectors.

The following theorem is proved in [55]: 
Theorem 4.1. Let a function $\Phi: \Omega \longrightarrow \mathbb{F}$ be continuous in a domain $\Omega \subset E_{3}$ and the functions $U_{k}: \Omega \longrightarrow \mathbb{R}$ from the expansion

$$
\Phi\left(x e_{1}+y e_{2}+z e_{3}\right)=\sum_{k=1}^{\infty} U_{k}(x, y, z) e_{k},
$$

be $\mathbb{R}$-differentiable in $\Omega$. In order that the function $\Phi$ be monogenic in $\Omega$, it is necessary and sufficient that the conditions (3.3) be satisfied and the following relations be fulfilled in $\Omega$ :

$$
\begin{gathered}
\sum_{k=1}^{\infty}\left|\frac{\partial U_{k}(x, y, z)}{\partial x}\right|<\infty \\
\lim _{\varepsilon \rightarrow 0+0} \sum_{k=1}^{\infty} \mid U_{k}\left(x+\varepsilon h_{1}, y+\varepsilon h_{2}, z+\varepsilon h_{3}\right)-U_{k}(x, y, z) \\
-\frac{\partial U_{k}(x, y, z)}{\partial x} \varepsilon h_{1}-\frac{\partial U_{k}(x, y, z)}{\partial y} \varepsilon h_{2} \\
-\frac{\partial U_{k}(x, y, z)}{\partial z} \varepsilon h_{3} \mid \varepsilon^{-1}=0 \quad \forall h_{1}, h_{2}, h_{3} \in \mathbb{R} .
\end{gathered}
$$

Note that the relations (4.2), (4.3) are conditioned by the infinite dimensionality of the algebra $\mathbb{F}$.

In the papers $[45,55]$ we established that any spherical function of order $n$ is a component of the monogenic function $\Phi(\zeta)=a \zeta^{n}$, where $\zeta=x e_{1}+y e_{2}+z e_{3}$ and the coefficient $a \in \mathbb{F}$ is found explicitly.

In [66], we inserted the algebra $\mathbb{F}$ in the topological vector space $\widetilde{\mathbb{F}}:=$ $\left\{g=\sum_{k=1}^{\infty} c_{k} e_{k}: c_{k} \in \mathbb{R}\right\}$ with the topology of coordinate-wise convergence.

For functions taking values in $\widetilde{\mathbb{F}}$, the formulation of statement similar to Theorem 4.2 can be simplified (see [66]):

Theorem 4.2. Let in the expansion (4.1) of a function $\Phi: \Omega \longrightarrow \widetilde{\mathbb{F}}$ the functions $U_{k}: \Omega \longrightarrow \mathbb{R}$ be $\mathbb{R}$-differentiable in $\Omega$. In order that the function $\Phi$ be monogenic in $\Omega$, it is necessary and sufficient that the conditions (3.3) be satisfied in $\Omega$.

The relation between monogenic functions taking values in $\widetilde{\mathbb{F}}$ and solutions of the three-dimensional Laplace equation is established in the following statement (cf. [66] as well as Theorem 1.20 in [55]):

Theorem 4.3. For every doubly continuously differentiable function $u$ : $\Omega \longrightarrow \mathbb{R}$ which satisfies Eq. (1.2) in a simply connected domain $\Omega \subset$ $\mathbb{R}^{3}$ there exists a monogenic function $\Phi: \Omega \longrightarrow \widetilde{\mathbb{F}}$ such that the first component of expansion (4.1) is $U_{1} \equiv u$. 
In [66] we considered also monogenic functions taking values in a topological vector space $\widetilde{\mathbb{G}}$ being an expansion of another infinite-dimensional commutative Banach algebra associated with the three-dimensional Laplace equation. For monogenic functions $\Phi: \Omega \longrightarrow \widetilde{\mathbb{G}}$ we established results similar to Theorems 4.2 and 4.3. And also, we described relations between monogenic functions taking values in either the space $\widetilde{\mathbb{F}}$ or the space $\widetilde{\mathbb{G}}$ and solutions of system (1.1).

Essentially, P. W. Ketchum [27] considered the space $\widetilde{\mathbb{F}}$ and M. N. Roşculet [79] considered the space $\widetilde{G}$ though they did not use the notion of topological vector space as well as the differentiability in the sense of Gâteaux.

In the paper [68] we construct explicitly principal extensions (3.5) of analytic functions of a complex variable into a complexification $\mathbb{F}_{\mathbb{C}}$ of the algebra $\mathbb{F}$. And also, we proved that any monogenic function $\Phi: \Omega \longrightarrow \widetilde{\mathbb{F}}_{\mathbb{C}}$ taking values in a complexification $\widetilde{\mathbb{F}}_{\mathbb{C}}$ of the space $\widetilde{\mathbb{F}}$ can be extended to a monogenic function given in a certain domain of the greater dimension than the domain $\Omega \subset E_{3}$.

In [69] we proved integral theorems for monogenic functions taking values in either the algebra $\mathbb{F}_{\mathbb{C}}$ or the topological vector space $\widetilde{\mathbb{F}}_{\mathbb{C}}$.

\subsection{An infinite-dimensional vector space associated with axial-symmetric potential fields}

In the case where a spatial potential field is symmetric with respect to the axis $O x$, a potential function $u(x, y, z)$ satisfying Eq. (1.2) is also symmetric with respect to the axis $O x$, i.e. $u(x, y, z)=\varphi(x, r)=$ $\varphi(x,-r)$, where $r:=\sqrt{y^{2}+z^{2}}$, and $\varphi$ is known as the axial-symmetric potential. Then in a meridian plane $x O r$ there exists a function $\psi(x, r)$ known as the Stokes flow function such that the functions $\varphi$ and $\psi$ satisfy the following system of equations degenerating on the axis $O x$ :

$$
r \frac{\partial \varphi(x, r)}{\partial x}=\frac{\partial \psi(x, r)}{\partial r}, \quad r \frac{\partial \varphi(x, r)}{\partial r}=-\frac{\partial \psi(x, r)}{\partial x}
$$

Let $\mathbb{H}_{\mathbb{C}}:=\left\{a=\sum_{k=1}^{\infty} a_{k} e_{k}: a_{k} \in \mathbb{C}, \sum_{k=1}^{\infty}\left|a_{k}\right|<\infty\right\}$ be a commutative associative Banach algebra over the complex field with the norm $\|a\|_{\mathbb{H}_{\mathbb{C}}}:=$ $\sum_{k=1}^{\infty}\left|a_{k}\right|$ and the following multiplication table for elements of the basis $\left\{e_{k}\right\}_{k=1}^{\infty}$ :

$$
e_{n} e_{1}=e_{n}, \quad e_{m} e_{n}=\frac{1}{2}\left(e_{m+n-1}+(-1)^{n-1} e_{m-n+1}\right) \quad \forall m \geq n \geq 1
$$

that was offered by I. P. Mel'nichenko (cf., e.g., [43, 45]). 
The set $\mathfrak{I}:=\left\{g \in \mathbb{H}_{\mathbb{C}}: \sum_{k=1}^{\infty}(-1)^{k}\left(\operatorname{Re} c_{2 k-1}-\operatorname{Im} c_{2 k}\right)=0\right.$, $\left.\sum_{k=1}^{\infty}(-1)^{k}\left(\operatorname{Re} c_{2 k}+\operatorname{Im} c_{2 k-1}\right)=0\right\}$ is a maximal ideal of the algebra $\mathbb{H}_{\mathbb{C}}$.

Consider the plane $\mu_{e_{1}, e_{2}}:=\left\{\zeta=x e_{1}+r e_{2}: x, r \in \mathbb{R}\right\}$. We use the denotation $G$ for congruent domains in $\mathbb{R}^{2}, \mu_{e_{1}, e_{2}}$ and $\mathbb{C}$.

A continuous function $\Phi: G \longrightarrow \mathbb{H}_{\mathbb{C}}$ is monogenic in a domain $G$ if for every $\zeta \in G$ there exists an element $\Phi^{\prime}(\zeta) \in \mathbb{H}_{\mathbb{C}}$ such that the equality (3.2) is fulfilled for all $h \in \mu_{e_{1}, e_{2}}$. A theorem similar to Theorem 4.1 is true for functions $\Phi: G \longrightarrow \mathbb{H}_{\mathbb{C}}$ (see [55]).

In what follows, a domain $G$ of a meridian plane $x O r$ is symmetric with respect to the axis $O x$.

Theorem 4.4 ( $[43,45])$. If a domain $G$ of a meridian plane $x O r$ is symmetric with respect to the axis $O x$ and convex in the direction of axis Or, then every monogenic function $\Phi: G \longrightarrow \mathbb{H}_{\mathbb{C}}$ can be expressed in the form

$$
\Phi(\zeta)=\frac{1}{2 \pi i} \int_{\Gamma_{\zeta}}(A \Phi)(t)\left(t e_{1}-\zeta\right)^{-1} d t+\Phi_{0}(\zeta) \quad \forall \zeta=x e_{1}+r e_{2} \in G,
$$

where $\Phi_{0}: G \longrightarrow \mathfrak{I}$ is a monogenic function taking values in the ideal $\mathfrak{I}$.

In the papers $[43,45]$, for every function $F: G \longrightarrow \mathbb{C}$ analytic in such a domain of the complex plane as it is considered in Theorem 4.4, we constructed explicitly the expansion of principal extension (3.5) with respect to the basis:

$$
\frac{1}{2 \pi i} \int_{\Gamma_{\zeta}}\left(t e_{1}-\zeta\right)^{-1} F(t) d t=U_{1}(x, r) e_{1}+2 \sum_{k=2}^{\infty} U_{k}(x, r) e_{k}
$$

where $\quad U_{k}(x, r):=\frac{1}{2 \pi i} \int_{\Gamma_{\zeta}} \frac{F(t)}{\sqrt{(t-z)(t-\bar{z})}}\left(\frac{\sqrt{(t-z)(t-\bar{z})}-(t-x)}{r}\right)^{k-1} d t$ for $k=$ $1,2, \ldots, \zeta=x e_{1}+r e_{2}, z=x+i r$, and the curve $\Gamma_{\zeta}$ is an arbitrary closed Jordan rectifiable curve in $G$ that embraces the segment connecting the points $z$ and $\bar{z}$, and $\sqrt{(t-z)(t-\bar{z})}$ is a continuous branch of this function analytic with respect to $t$ outside of the mentioned segment in the case $\operatorname{Im} z \neq 0$, and we define $\sqrt{(t-z)(t-\bar{z})}:=t-z$ for each $z \in G$ with $\operatorname{Im} z=0$.

We described relations between principal extensions of analytic functions into the plane $\mu_{e_{1}, e_{2}}$ and solutions of system (4.4), viz.: 
Theorem 4.5 ([43,45]). If $F: G \longrightarrow \mathbb{C}$ is an analytic function in such a domain of the complex plane as it is considered in Theorem 4.4, then the first and the second components of principal extension (4.5) generate solutions $\varphi$ and $\psi$ of system (4.4) in $G$ by the formulas

$$
\varphi(x, r)=U_{1}(x, r), \quad \psi(x, r)=r U_{2}(x, r) .
$$

It follows from the relations (4.5) and (4.6) that the functions

$$
\begin{gathered}
\varphi(x, r)=\frac{1}{2 \pi i} \int_{\Gamma_{\zeta}} \frac{F(t)}{\sqrt{(t-z)(t-\bar{z})}} d t, \\
\psi(x, r)=-\frac{1}{2 \pi i} \int_{\Gamma_{\zeta}} \frac{F(t)(t-x)}{\sqrt{(t-z)(t-\bar{z})}} d t, \quad z=x+i r,
\end{gathered}
$$

are solutions of system (4.4) in the domain $G$.

In the papers $[45,50]$ we generalized integral expressions (4.7) and (4.8) for the axial-symmetric potential $\varphi$ and the Stokes flow function $\psi$, respectively, to the case of arbitrary simply connected domain symmetric with respect to the axis $O x$. In [58], we shown that in this case the functions $\varphi$ and $\psi$ are expressed by the formulas (4.6) via components of principal extensions (4.5) taking values in an infinite-dimensional topological vector space $\widetilde{\mathbb{H}}_{\mathbb{C}}:=\left\{g=\sum_{k=1}^{\infty} c_{k} e_{k}: c_{k} \in \mathbb{C}\right\}$ with the topology of coordinate-wise convergence (see also [56]). In [56], we proved integral theorems for monogenic functions taking values in either the algebra $\mathbb{H}_{\mathbb{C}}$ or the vector space $\widetilde{\mathbb{H}}_{\mathbb{C}}$.

In the papers $[45,50]$ we established sufficient conditions for continuous continuations of the functions (4.7), (4.8) on the boundary $\partial G$ of a domain $G$ and obtained estimations for modules of continuity of boundary values of the mentioned functions.

In [11], we established an integral expression of generalized axialsymmetric potential that is a generalization of integral expressions obtained by A. G. Mackie [39], P. Henrici [23], Yu. P. Krivenkov [33] and G. N. Polozhii [73].

In the papers $[45,51-54]$ we proved that all axial-symmetric potentials and Stokes flow functions, i.e. solutions of system (4.4) in $G$ with a physical interpretation, are represented by the integral expressions (4.7) and (4.8), respectively.

Using these integral expressions, we developed methods for solving BVPs in a meridian plane of spatial axial-symmetric potential field 
(see $[45,51-54])$. In addition, using the integral expression (4.8) for the Stokes flow function, in the papers $[44,45,58]$ we obtained some results having a natural physical interpretation. Namely, for a BVP with respect to a steady streamline of the ideal incompressible fluid along an axial-symmetric body, we obtained criteria of solvability by means distributions of sources and dipoles on the axis of symmetry and constructed unknown solutions using multipoles together with dipoles distributed on the axis.

\section{References}

[1] H. A. v. Beckh-Widmanstetter, Läßt sich die eigenschaft der analytischen funktionen einer gemeinen komplexen veränderlichen, potentiale als bestandteile zu liefern, auf zahlsysteme mit drei einheiten verallgemeinern? // Monatshefte für Mathematik und Physik, 23 (1912), 257-260.

[2] E. K. Blum, A theory of analytic functions in Banach algebras // Trans. Amer. Math. Soc., 78 (1955), 343-370.

[3] D. Boccaletti, F. Catoni, R. Cannatay, V. Catoniz, E. Nichelattix, P. Zampetti, The mathematics of Minkowski space-time and an introduction to ccmmutative hypercomplex numbers, Springer, 2006.

[4] E. Cartan, Les groupes bilinéares et les systèmes de nombres complexes // Annales de la faculté des sciences de Toulouse, 12 (1898), N 1, 1-64.

[5] F. Colombo, I. Sabadini, D. C. Struppa, Noncommutative functional calculus: theory and applications of slice hyperholomorphic functions, Progress in Mathematics, 289, Birkhäuser Basel, 2011.

[6] A. Douglis, A function-theoretic approach to elliptic systems of equations in two variables // Communications on Pure and Applied Mathematics, 6 (1953), N 2, 259-289.

[7] R. Fueter, Die funktionentheorie der differentialgleichungen $\Delta u=0$ und $\Delta \Delta u=$ 0 mit vier reellen variablen // Comment. math. helv., 7 (1935), 307-330.

[8] S. V. Gryshchuk, $\mathbb{B}$-valued monogenic functions and their applications to boundary value problems in displacements of 2-D Elasticity // Analytic Methods of Analysis and Differential Equations: AMADE 2015, Cambridge Scientic Publishers, Cottenham, (2016), 37-47.

[9] S. V. Gryshchuk, One-dimensionality of the kernel of the system of Fredholm integral equations for a homogeneous biharmonic problem // Zb. Pr. Inst. Mat. NAN Ukr., 14 (2017), N 1, 128-139 (in Ukrainian).

[10] S. V. Gryshchuk, Commutative complex algebras of the second rank with unity and some cases of plane orthotropy. I, II // Ukr. Mat. Zh., 70 (2018), N 8, 1058-1071; N 10, 1382-1389 (in Ukrainian). 
[11] S. V. Grishchuk, S. A. Plaksa, Integral representations of generalazid axially symmetric potentials in a simply connected domain // Ukr. Math. J., 61 (2009), N 2, 195-213.

[12] S. V. Grishchuk, S. A. Plaksa, Monogenic functions in a biharmonic algebra // Ukr. Math. J., 61 (2009), N 12, 1865-1876.

[13] S. V. Gryshchuk, S. A. Plaksa, On the logarithmic residues of monogenic functions of biharmonic variable // Zb. Pr. Inst. Mat. NAN Ukr., 7 (2010), N 2, 227-234 (in Russian).

[14] S. V. Gryshchuk, S. A. Plaksa, Basic properties of monogenic functions in a biharmonic plane // Complex Analysis and Dynamical Systems V, Contemporary Mathematics, 591 (2013), Amer. Math. Soc., Providence, RI, 127-134.

[15] S. V. Gryshchuk, S. A. Plaksa, Schwarz-type integrals in a biharmonic plane // Intern. J. of Pure and Appl. Math., 83 (2013), N 1, 193-211.

[16] S. V. Gryshchuk, S. A. Plaksa, Monogenic functions in the biharmonic boundary value problem // Mathematical Methods in the Applied Sciences, 39 (2016), N 11, 2939-2952.

[17] S. V. Gryshchuk, S. A. Plaksa, A Schwartz-type boundary value problem in a biharmonic plane // Lobachevskii Journal of Mathematics, 38 (2017), N 3, 435-442 .

[18] S. V. Gryshchuk, S. A. Plaksa, Reduction of a Schwartz-type boundary value problem for biharmonic monogenic functions to Fredholm integral equations // Open Mathematics, 15 (2017), N 1, 374--381.

[19] S. V. Gryshchuk, S. A. Plaksa, Schwartz-type boundary value problems for monogenic functions in a biharmonic algebra // Analysis as a Life. Trends in Mathematics, Birkhauser, Cham, (2019), 193-211.

[20] K. Gürlebeck, W. Sprössig, Quaternionic and Clifford calculus for physicists and engineers, John Wiley and Sons, 1997.

[21] E. Goursat, Cours d'analyse mathematique, 2, Gauthier-Villars, Paris, 1910.

[22] W. Hamilton, Elements of quaternions, University of Dublin Press, 1866.

[23] P. Henrici, On the domain of regularity of generalized axially symmetric potentials // Proc. Amer. Math. Soc., 8 (1957), N 1, 29-31.

[24] O. F. Herus, On the Cauchy theorem for hyperholomorphic functions of spatial variable // J. Math. Sci., 229 (2018), N 1, 1-6.

[25] E. Hille, R. S. Phillips, Functional analysis and semi-groups, Providence, R.I.: Amer. Math. Soc., 1957.

[26] P. W. Ketchum, Analytic functions of hypercomplex variables // Trans. Amer. Math. Soc., 30 (1928), 641-667. 
[27] P. W. Ketchum, A complete solution of Laplace's equation by an infinite hypervariable // Amer. J. Math., 51 (1929), 179-188.

[28] V. V. Kisil, Erlangen programme at large: an overview // Advances in Applied Analysis, Trends in Mathematics. Springer, Basel, (2012), 1-94.

[29] V. F. Kovalev, Biharmonic Schwarz problem, Preprint 86.16, Kiev: Inst. of Math. of NAS of Ukraine, 1986 (in Russian).

[30] V. F. Kovalev, I. P. Mel'nichenko, Biharmonic functions on biharmonic plane // Dop. AN Ukr. Ser. A., (1981), N 8 , 25-27 (in Russian).

[31] V. F. Kovalev, I. P. Mel'nichencko, Algebras of functional-invariant solutions of the p-biharmonic equation, Preprint 91.10, Kiev: Inst. of Math. of NAS of Ukraine, 1991 (in Russian).

[32] V. V. Kravchenko, M. V. Shapiro, Integral representations for spatial models of mathematical physics, Pitman Research Notes in Mathematics, Addison Wesley Longman Inc, 1996.

[33] Yu. P. Krivenkov, Representation of solutions of the Euler-Poisson-Darboux equation via analytic functions // Dokl. Akad. Nauk SSSR, 116 (1957), N 4, 545-548.

[34] K. S. Kunz, Application of an algebraic technique to the solution of Laplace's equation in three dimensions // SIAM J. Appl. Math., 21 (1971), N 3, 425-441.

[35] T. S. Kuzmenko, V. S. Shpakivskyi, Generalized integral theorems for the quaternionic G-monogenic mappings // J. Math. Sci., 224 (2017), N 4, 530-540

[36] H. Leutwiler, Modified quaternionic analysis in $\mathbb{R}^{3} / /$ Complex variables theory appl., 20 (1992), 19-51.

[37] E. R. Lorch, The theory of analytic function in normed abelin vector rings // Trans. Amer. Math. Soc., 54 (1943), 414-425.

[38] M. E. Luna-Elizarrarás, M. Shapiro, D. C. Struppa, A. Vajiac, Bicomplex holomorphic functions: the algebra, geometry and analysis of bicomplex numbers, Birkhäuser, 2015.

[39] A. G. Mackie, Contour integral solutions of a class of differential equations // J. Ration. Mech. Anal., 4 (1955), N 5, 733-750.

[40] I. P. Mel'nichenko, The representation of harmonic mappings by monogenic functions // Ukr. Math. J., 27 (1975), N 5, 499-505.

[41] I. P. Mel'nichenko, Biharmonic bases in algebras of the second rank // Ukr. Math. J., 38 (1986), N 2, 224-226.

[42] I. P. Mel'nichenko, Algebras of functionally invariant solutions of the threedimensional Laplace equation // Ukr. Math. J., 55 (2003), N 9, 1551-1557. 
[43] I. P. Mel'nichenko, S.A. Plaksa, Potential fields with axial symmetry and algebras of monogenic functions of vector variable. I, II, III // Ukr. Math. J., 49 (1996), N 11, 1717-1730; N 12, 1916-1926; 49 (1997), N 2, 253-268.

[44] I. P. Mel'nichenko, S. A. Plaksa, Outer boundary problems for the Stokes flow function and steady streamline along axial-symmetric bodies // Complex Analysis and Potential Theory, Kiev, Institute of Mathematics of the National Academy of Sciences of Ukraine, (2003), 82-91.

[45] I. P. Mel'nichenko, S. A. Plaksa, Commutative algebras and spatial potential fields, Kiev: Inst. of Math. of NAS of Ukraine, 2008 (in Russian).

[46] S. G. Mikhlin, The plane problem of the theory of elasticity, Trans. Inst. of seismology, Acad. Sci. USSR., N 65, Moscow-Leningrad, 1935 (in Russian).

[47] G. C. Moisil, N. Theodoresco, Functions holomorphes dans l'espace // Mathematica (Cluj), 5 (1931), 142-159.

[48] N. I. Muskhelishvili, Some basic problems of the mathematical theory of elasticity, Noordhoff International Publishing, Leiden, 1977.

[49] B. Peirce, Linear associative algebra // American Journal of Mathematics, 7 (1881), N 1, 97-229.

[50] S. A. Plaksa, On integral representations of an axisymmetric potential and the Stokes flow function in domains of the meridian plane. I, II // Ukr. Math. J., 53 (2001), N 5, 726-743; N 6, 938-950.

[51] S. A. Plaksa, Dirichlet problem for an axisymmetric potential in a simply connected domain of the meridian plane // Ukr. Math. J., 53 (2001), N 12, 19761997.

[52] S. A. Plaksa, On an outer Dirichlet problem solving for the axial-symmetric potential // Ukr. Math. J., 54 (2002), N 12, 1634-1641.

[53] S. A. Plaksa, Dirichlet problem for the Stokes flow function in a simply connected domain of the meridian plane // Ukr. Math. J., 55 (2003), N 2, 197-231.

[54] S. Plaksa, Singular and Fredholm integral equations for Dirichlet boundary problems for axial-symmetric potential fields // Factotization, Singular Operators and Related Problems: Proc. of the Conference in Honour of Professor Georgii Litvinchuk, Funchal, January 28-February 1, 2002, Kluwer Academic Publishers, (2003), 219-235.

[55] S. A. Plaksa, Commutative algebras associated with classic equations of mathematical physics // Advances in Applied Analysis, Trends in Mathematics, Springer, Basel, (2012), 177-223.

[56] S. A. Plaksa, Integral theorems for monogenic functions in an infinite-dimensional space with a commutative multiplication // Zb. Pr. Inst. Mat. NAN Ukr., 10 (2013), N 4-5, 306-319. 
[57] S. A. Plaksa, On differentiable and monogenic functions in a harmonic algebra // Zb. Pr. Inst. Mat. NAN Ukr., 14 (2017), N 1, 210-221.

[58] S. A. Plaksa, Axial-symmetric potential flows // Models and Theories in Social Systems, Springer International Publishing, (2019), 165-195.

[59] S. A. Plaksa, S. V. Gryshchuk, V. S. Shpakivskyi, Commutative algebras of monogenic functions associated with classic equations of mathematical physic // Complex Analysis and Dynamical Systems IV, Contemporary Mathematics, 553 (2011), Amer. Math. Soc., Providence, RI, 245-258.

[60] S. A. Plaksa, R. P. Pukhtaievych, Constructive description of monogenic functions in a three-dimensional harmonic algebra with one-dimensional radical // Ukr. Math. J., 65 (2013), N 5, 740-751.

[61] S. Plaksa, R. Pukhtaievych, Monogenic functions in three-dimensional harmonic commutative algebras // Complex analysis and potential theory with applications, Cambridge Scientific Publishers, (2014), 147-155.

[62] S. A. Plaksa, R. P. Pukhtaievych, Monogenic functions in a finite-dimensional semi-simple commutative algebra // An. St. Univ. Ovidius Constanta, 22 (2014), N 1, 221-235.

[63] S. A. Plaksa, V. S. Shpakivskyi, Limiting values of the Cauchy type integral in a three-dimensional harmonic algebra // Eurasian Math. J., 3 (2012), N 2, 120-128.

[64] S. A. Plaksa, V. S. Shpakivskyi, On limiting values of Cauchy type integral in a harmonic algebra with two-dimensional radical // Annales Universitatis Mariae Curie-Sklodowska. Sectio A., 67 (2013), N 1, 57-64.

[65] S. A. Plaksa, V. S. Shpakivskyi, Monogenic functions in a finite-dimensional algebra with unit and radical of maximal dimensionality // Journal of Algerian Mathematical Society, 1 (2014), N 1, 1-13.

[66] S. A. Plaksa, V. S. Shpakivskyi, A description of spatial potential fields by means of monogenic functions in infinite-dimensional spaces with a commutative multiplication // Bull. Soc. Sci. Lett. Łódź, Ser. Rech. Déform., 62 (2012), N 2, 55-65.

[67] S. A. Plaksa, V. S. Shpakivskyi, Cauchy theorem for a surface integral in commutative algebras // Complex Variables and Elliptic Equations, 59 (2014), N 1, 110-119.

[68] S. A. Plaksa, V. S. Shpakivskyi, An extension of monogenic functions and spatial potentials // Lobachevskii J. Math., 38 (2017), N 2, 330-337.

[69] S. A. Plaksa, V. S. Shpakivskyi, Integral theorems for monogenic functions in an infinite-dimensional space with a commutative multiplication // Bull. Soc. Sci. Lett. Łódź, Ser. Rech. Déform., 68 (2018), N 2, 25-36.

[70] S. A. Plaksa, V. S. Shpakovskii, Constructive description of monogenic functions in a harmonic algebra of the third rank // Ukr. Math. J., 62, (2011), N 8, 12511266. 
[71] S.A. Plaksa, V.S. Shpakovskii, On the logarithmic residues of monogenic functions in a three-dimensional harmonic algebra with two-dimensional radical // Ukr. Math. J., 65 (2013), N 7, 1079-1086.

[72] A. Pogorui, R. M. Rodriguez-Dagnino, M. Shapiro, Solutions for PDEs with constant coefficients and derivability of functions ranged in commutative algebras // Math. Meth. Appl. Sci., 37(17) (2014), 2799-2810.

[73] G. N. Polozhii, Theory and application of p-analytic and $(p, q)$-analytic functions, Kiev, Naukova Dumka, 1973 (in Russian).

[74] G. B. Price, An Introduction to multicomplex spaces and functions, Marcel Dekker, New York, 1991.

[75] R. P. Pukhtaievych, Power series and Laurent series in three-dimensional harmonic algebra with a one-dimensional radical // Zb. Pr. Inst. Mat. NAN Ukr., 9 (2012), N 2, 311-326 (in Russian).

[76] R. P. Pukhtaievych, Monogenic functions in a three-dimensional harmonic semisimple algebra // Zb. Pr. Inst. Mat. NAN Ukr., 10 (2013), N 4-5, 352-361.

[77] R. Pukhtaievych, S. Plaksa, On logarithmic residue of monogenic functions in a three-dimensional commutative algebra with one-dimensional radical // An. St. Ovidius Constanta, 25 (2017), N 3, 167-182.

[78] F. Ringleb, Beiträge zur funktionentheorie in hyperkomplexen systemen // Rend. Circ. Mat. Palermo., 57 (1933), 311-340.

[79] M. N. Roşculeţ, Algebre infinite associate la ecuaţii cu derivate parţiale, omogene, cu coeficienţi constanţi de ordin oarecare // Studii şi Cercetări Matematice, 6 (1955), N 3-4 , 567-643 (in Romanian).

[80] J. Ryan, Dirac operators, conformal transformations and aspects of classical harmonic analysis // J. of Lie Theory, 8 (1998), 67-82.

[81] G. Scheffers, Verallgemeinerung der grundlagen der gewöhnlich complexen fuktionen, I, II // Ber. Verh. Sachs. Akad. Wiss. Leipzig Mat.-Phys. Kl., 45 (1893), 828--848; 46 (1894), 120--134.

[82] C. Segre, The real representations of complex elements and extentions to bicomlex systems // Math. Ann., 40 (1892), 413-467.

[83] V. S. Shpakivskyi, Power and Laurent series in a three-dimensional harmonic algebra // Zb. Pr. Inst. Mat. NAN Ukr., 7 (2010), N 2, 314-321 (in Russian).

[84] V. S. Shpakivskyi, Monogenic functions in finite-dimensional commutative associative algebras // Zb. Pr. Inst. Mat. NAN Ukr., 12 (2015), N 3, 251-268.

[85] V. Shpakivskyi, Constructive description of monogenic functions in a finitedimensional commutative associative algebra // Adv. Pure Appl. Math., 7 (2016), N $1,63-75$. 
[86] V. S. Shpakivskyi, Curvilinear integral theorems for monogenic functions in commutative associative algebras // Adv. Appl. Clifford Algebras, 26 (2016), 417-434.

[87] V. S. Shpakivskyi, Hypercomplex functions and exact solutions of one hydrodynamic equation // Zb. Pr. Inst. Mat. NAN Ukr., 14 (2017), N 1, 262-274 (in Russian).

[88] V. S. Shpakivskyi, On monogenic functions defined in different commutative algebras // J. Math. Sci., 239 (2019), N 1, 92-109.

[89] V. S. Shpakivskyi, On monogenic functions on expansions of a commutative algebra // Proc. Intern. Geometry Center, 11 (2018), N 3, 1-18 (in Ukrainian).

[90] V. S. Shpakivskyi, Hypercomplex method for solving linear PDEs with constant coefficients // Proc. of IAMM of NASU, 32 (2018), 147-168 (in Ukrainian).

[91] V. S. Shpakivs'kyi, T.S. Kuz'menko, On one class of quaternionic mappings // Ukr. Math. J., 68 (2016), N 1, 127-143.

[92] V. S. Shpakivskyi, T. S. Kuzmenko, Integral theorems for the quaternionic Gmonogenic mappings // An. Şt. Univ. Ovidius Constanţa, 24 (2016), N 2, 271281.

[93] V. S. Shpakivskyi, T. S. Kuzmenko, On monogenic mappings of a quaternionic variable // J. Math. Sci., 221 (2017), N 5, 712-726.

[94] V. S. Shpakivskyi, S. A. Plaksa, Integral theorems in a commutative threedimensional harmonic algebra // Progress in Analysis and its Applications: Proc. of the 7th Intern. ISAAC Congress, Imperial College, London, UK, 13-18 July 2009, World Scientific, London, (2010), 232-239.

[95] V. S. Shpakivskyi, S. A. Plaksa, Integral theorems and a Cauchy formula in a commutative three-dimensional harmonic algebra // Bulletin Soc. Sci. et Lettr. Lódź, 60 (2010), 47-54.

[96] V. I. Smirnov, A course of higher mathematics, 3, Part 2, Pergamon Press, Oxford, 1964.

[97] L. Sobrero, Nuovo metodo per lo studio dei problemi di elasticità, con applicazione al problema della piastra forata // Ricerche di Ingegneria, 13 (1934), N 2, 255264.

[98] E. Study, Uber systeme complexer zahlen und ihre anwendung in der theorie der transformationsgruppen // Monatshefte für Mathematik und Physik, 1 (1890), 283-354.

[99] A. Sudbery, Quaternionic analysis // Math. Proc. Camb. Phil. Soc. 85 (1979), 199-225.

[100] G. Tolstoff, Sur les fonctions bornées vérifiant les conditions de CauchyRiemann // Rec. Math. [Mat. Sbornik] N.S., 10(52) (1942), N 1-2, 79-85. 
[101] Ju. Ju. Trokhimchuk, Continuous mappings and conditions of monogeneity, Israel Program for Scientific Translations, Jerusalem., Daniel Davey \& Co. Inc., New York, 1964.

\section{CONTACT INFORMATION}

Sergiy A. Plaksa Department of Complex Analysis and Potential Theory, Institute of Mathematics of the National Academy of Science of Ukraine, Kyiv, Ukraine

E-Mail: plaksa62@gmail.com 